\title{
Correlación entre estado nutricional y creatividad de estudiantes de la comunidad Awajún de Chapi, Condorcanqui, 2017.
}

\section{Correlation between nutritional status and creativity of students of the community Awajún de Chapi, Condorcanqui, 2017}

\author{
José D. Farje Escobedo ${ }^{1}$, Mirtha Chuquizuta Culquimboz ${ }^{2}$ Humberto Saukai Macahuachi ${ }^{3}$
}

\begin{abstract}
RESUMEN
El objetivo del trabajo fue determinar la correlación entre el estado nutricional y la creatividad de los estudiantes Awajún de la comunidad nativa de Chapi, distrito de Nieva, Amazonas, comprendidos entre ocho y doce años de edad. La investigación fue de tipo descriptivo correlacional (Hernández, Fernández y Baptista, 2010). La muestra fue de tipo no probabilística, conformada por 30 estudiantes que cursan tercero, cuarto, quinto y sexto grado de educación primaria. Para la recolección de datos, se emplearon dos instrumentos: La tabla de valoración nutricional antropométrica validada por el Ministerio de Salud; y la prueba de imaginación creativa - PIC, validada por TEAediciones, Madrid, España. Los resultados evidencian que el $60 \%$ del total de la muestra, de estudiantes tienen desnutrición crónica y solamente el $40 \%$ están en un estado normal de nutrición; en cuanto a la creatividad general, alcanzan una media aritmética centil de 9 que es muy deficiente. La correlación entre el estado nutricional y la creatividad de los estudiantes muestran que no existe correlación directa significativa, sin embargo relacionando la creatividad general y grado de estudios se evidencia que existe una correlación, lo que significa que conforme avanzan en su grado de estudios se incrementa levemente su creatividad.
\end{abstract}

Palabras clave: Desnutrición, creatividad

\begin{abstract}
The objective of the work was to determine the correlation between the nutritional status and creativity of the Awajún students of the native community of Chapi, district of Nieva, Amazonas, between eight and twelve years of age. The investigation was of descriptive correlational type (Hernández, Fernández and Baptista, 2010). The sample was of a non-probabilistic type, made up of 30 students who study third, fourth, fifth and sixth grade of primary education. For the collection of data, two instruments were used: The anthropometric nutritional assessment table validated by the Ministry of Health; and the creative imagination test - PIC, validated by TEAediciones, Madrid, Spain. The results show that $60 \%$ of the total sample, of students have chronic malnutrition and only $40 \%$ are in a normal state of nutrition; As for general creativity, they reach a centile arithmetic mean of 9 which is very deficient. The correlation between the nutritional status and the creativity of the students show that there is no significant direct correlation, however, relating the general creativity and the degree of studies, it is evidenced that there is a correlation, which means that as they advance in their degree of studies they increase slightly his creativity.
\end{abstract}

Keywords: Malnutrition, creativity.

${ }^{1}$ UniversidadNacional Toribio Rodríguez de Mendoza de Amazonas, Facultad de Educación y Ciencias de la Comunicación, Escuela Profesional de Educación. Correo:jose.farje@untrm.edu.pe

${ }^{2}$ Institución Educativa María Auxiliadora, Chachapoyas.

${ }^{3}$ UniversidadNacional Toribio Rodríguez de Mendoza de Amazonas, Facultad de Educación y Ciencias de la Comunicación, Escuela Profesional de Educación Intercultural Bilingüe. 


\section{INTRODUCCIÓN}

Dando un vistazo al mundo actual, tenemos que enfrentarnos a una verdad incómoda pero innegable, que en particular se resume: las vidas de millones de niños están malogradas por el solo hecho de haber nacido en un país, en una comunidad, en determinadas circunstancias. En todo el mundo hay más de 500 millones de niños que viven en zonas donde las inundaciones son extremadamente frecuentes, y cerca de 160 millones viven en zonas donde las sequias son de carácter grave o muy grave. La Organización Mundial de la Salud prevé que hasta el 2030, cada año se producirán cerca de 250.000 muertes más como consecuencia de la desnutrición, el paludismo, la diarrea y el estrés térmico, atribuible solamente al cambio climático (Unicef, 2016, p. 5).

La desnutrición está referida a un estado patológico ocasionado por la falta de absorción de nutrientes. "El estado nutricional de una persona es la resultante del equilibrio entre la ingesta de alimentos y sus requerimientos de nutrientes (..), el aprovechamiento de los nutrientes ingeridos depende de diversos factores como la combinación de alimentos o las condiciones del sistema gastrointestinal que los recibe" (Segura, et al. 2002, p. 8).

En el mundo en desarrollo, cerca de 200 millones de niños menores de 5 años sufren desnutrición crónica, cuyos efectos se harán sentir durante el resto de sus vidas. Además, el 13\% de los niños menores de 5 años padece desnutrición aguda, requiere tratamiento inmediato y atención médica urgente (Unicef, 2011, p. 4).

Continuando con el problema de la desnutrición, Segura et al. (2002), refiere:

Los requerimientos de nutrientes dependen del estado fisiológico de la persona. Los momentos de máxima demanda corresponden a aquellos de mayor reproducción celular, es decir los periodos de rápido crecimiento como la gestación, los primeros años de vida y la adolescencia. (...) Aquellas sociedades incapaces de asegurar un aporte suficiente de nutrientes para todos sus miembros y tienen una alta carga de morbilidad; son justamente aquellas que tienen los mayores problemas de estado nutricional infantil. La ingesta insuficiente y la enfermedad, se potencial mutuamente. (...) Los niños desnutridos tienen menos resistencia a las enfermedades, principalmente infecciosas, por lo que tienen mayor riesgo de caer enfermos. Las infecciones pueden causar pérdida de apetito, incremento del metabolismo, mala absorción intestinal por tránsito acelerado y reducción en las sales biliares y lesiones en la mucosa intestinal, todo lo cual empeora aún más el estado nutricional. (pág. 10-11).

El crecimiento económico del país en los últimos años viene reflejándose en una reducción sostenida de la pobreza. La tasa de desnutrición crónica infantil - DCI, en el promedio nacional, también se ha reducido de $31 \%$ en el año 2000 a $18.1 \%$, (MIDIS: ENDES, Encuesta demográfica y de salud familiar, 2012).

Los primeros años de vida constituyen la edad de oro en demanda de la atención alimentaria de los niños, si se ignora esa regla, entonces estamos descuidando a la población potencial que en un determinado momento contribuirán al desarrollo personal y social en un determinado contexto.

Particularmente, existen discrepancias con los datos precitados, dado que en la realidad se aprecia que la desnutrición está avanzando, independientemente del apoyo que reciben de los programas sociales impulsados por el gobierno, debido sobre todo a limitaciones que se presentan en los procesos de distribución y consumo de los alimentos, en paralelo con otras condiciones de salubridad presentes durante la ingesta.

En América Latina, entre las carencias básicas la desnutrición infantil crónica es un buen indicador o variable sustantiva, pues para que se dé de manera intensa en un territorio, tienden a concurrir una serie de privaciones que la potencian: baja educación de la familia, limitados ingresos, falta de acceso a amplias redes sociales y deficiencia en la atención sanitaria. Las mayores disparidades se encuentran en el Perú, Honduras, Guatemala y el Estado Plurinacional de Bolivia. Particularmente en Perú, donde la prevalencia de la desnutrición global es casi 9 veces mayor en niños y niñas de Huancavelica que entre los de Tacna; mientras que en Honduras la diferencia entre las zonas de mayor y menor prevalencias es de 35 puntos. Asimismo, mientras que en Guatemala casi el 75\% de los menores de la zona norte del país tienen un bajo peso para su edad, en la zona metropolitana se ve afectado el $41 \%$, y en el Estado Pluricultural de Bolivia, Santa Cruz tiene una prevalencia de $17.7 \%$, en tanto en Potosí afecta a la mitad de los menores de 5 años, mayores datos se encuentran en el cuadro 1 (Naciones Unidas CEPAL: La hora de la igualdad: Brechas por cerrar, caminos por abrir, 2010: 143).

Amazonas, es el departamento ubicado en noreste del país en la ceja de selva, está dividido en siete provincias y 83 distritos y tiene un área de $39249 \mathrm{~km}^{2}$. La cordillera del Cóndor atraviesa el territorio y es 
surcado por los ríos Marañón y sus afluentes, Santiago, Utcubamba, Cenepa y Nieva. La zona norte del departamento tiene un alto porcentaje de población indígena que vive en una serie de comunidades nativas ubicadas a la orilla de los ríos, por lo que el transporte es predominantemente fluvial, aunque también existen trochas y rutas carrozables. Los pueblos nativos de la Amazonía tienen una economía de subsistencia, se dedican en su mayoría a la pesca y la caza, aunque la deforestación de la selva haya afectado considerablemente estas actividades. La producción agraria está orientada sobre todo al cultivo del plátano y la yuca, destinados principalmente al autoconsumo y en segundo término a la venta de acopiadores de Chiclayo y Piura. (Instituto de estudios peruanos - Unicef, 2009: 17). El programa Juntos, pretende superar los niveles de desnutrición infantil, brindando apoyo a las personas más pobres, bajo ciertas condiciones, siendo el principal que los beneficiarios se comprometan a llevar a sus menores hijos al centro de salud y hacer asistir a sus hijos a la escuela; sin embargo la población Awajún consume más productos enlatados, ignorando muchas veces el consumo local, hay un gran incremento del producto foráneo, dado que la alimentación propia del lugar se limita a la yuca, el plátano y el pescado, y lo poco que les queda de dinero del programa, lo utilizan para comprar cosas nuevas provenientes de la costa, como atún, arroz y fideo; en consecuencia, el impacto del programa para superar la desnutrición de los niños, no es significativo. (Ídem, 34). Además, en su gran mayoría de escuelas se imparte la educación bilingüe intercultural en los tres niveles: inicial, primaria y secundaria El gobierno ofrece diversas las fuentes de financiamiento que el gobierno nacional asegura para implementar las estrategias e intervenciones efectivas para reducir la desnutrición crónica infantil - DCI. Según estimaciones del MIDIS - ENDES, 2013, entre el 2007 y el 2010, Amazonas tuvo una disminución del DCI de 12 puntos porcentuales; sin embargo, en el período 2010-2012 se incrementó en cuatro puntos, registrando $29.2 \%$; siendo los distritos que necesitan mayor atención, los de la provincia de Condorcanqui y el distrito de Imaza en Bagua. (MIDIS-ENDES, 2012, p. 140).

Existen tres tipos de desnutrición calórica: i) aguda, se presenta con una delgadez extrema, producto de una pérdida de peso asociada con períodos recientes de hambruna o enfermedad que se desarrolla muy rápidamente y es limitada en el tiempo, se mide como una deficiencia de peso por altura (P/A). ii) crónica, asociada normalmente a situaciones permanentes de ingesta insuficiente de alimentos o de frecuentes enfermedades, como ocurre en hogares con pobreza; se mide como retardo de altura para la edad (A/E). iii) global, es la combinación de las dos primeras, la cual se usa para dar seguimiento a los objetivos del milenio; se mide como deficiencia de peso para la $\operatorname{edad}(\mathrm{P} / \mathrm{AxA} / \mathrm{E}=\mathrm{P} / \mathrm{E})$. (Unicef, 2012).

La creatividad es una característica inherente al ser humano, susceptible de ser estimulada por el entorno personal, familiar y social del niño; al respecto, refiere Dacey, citado por Cemades (2008: 5) que en todo ser humano existe el impulso de experimentar, indagar, relacionar, en definitiva, de crear (...), el niño de educación infantil se encuentra en un período crítico donde se realiza el mayor desarrollo neuronal en el ser humano. Si en este período existen presiones externas autoritarias, el pensamiento creativo decrece. Asimismo, Gardner (1990), sostiene que existen relaciones entre inteligencia y creatividad llega a demostrar que al existir múltiples inteligencias, puede puntuarse alto en algunas de ellas y no en otras, y que, por tanto, su relación con la creatividad vendrá más por el tipo de inteligencia medida que por un constructo más abstracto de inteligencia (Penagos, 2002, Prieto, López y Fernández, 2003; citado por Fuentes, 2004, p. 43), además agrega que la creatividad se puede considerar desde: una característica de la persona, un proceso de la persona; como actividad cognitiva de dominio específico; de dominio general; como un producto; como un proceso o como parte del contexto. O simplemente de las cuatro "pes de la creatividad": persona, producto, proceso y presión-contexto.

De Prado (2001: 9) citado por Fuentes (2004), hace una taxonomía en la que distingue tres tipos de creatividad: a) creatividad objetiva o realista: referida a un tipo de creatividad relacionada con lo exterior, con lo que ya sabe y ha vivido la persona. Se traduce también en un cuestionamiento y planteamiento hipotético de la realidad para descubrir lo deficitario y negativo, y de esa forma que emerja una solución creativa a los problemas. b) creatividad imaginativa y fantástica, que emerge para sobrepasar los límites de la realidad, a través de un pensamiento analógico, imaginativo y fantástico-transformativo, alejados de lo real y sin ningún control lógico-racional. c) creatividad innovadora e inventiva, que proviene del pensamiento innovador, volcado al cambio y mejora de lo real, que se ajusta al deseo de la persona o a un ideal de excelencia. Busca lo mejor y asume que todo lo existente puede cambiar, no es fijo ni estático. Está relacionada con la invención o creación original de algo único e inexistente.

Teniendo en cuenta es realidad, la presente investigación se orienta a indagar la correlación entre el estado nutricional y la creatividad de estudiantes 
con edades que oscilan entre los 8 y 12 años de edad, de la comunidad Awajún de Chapi, Condorcanqui, durante el año, 2017.

\section{MATERIAL Y MÉTODO}

\section{Tipo y Diseño de la Investigación}

El tipo de investigación es descriptiva correlacional, de acuerdo con Hernández, Fernández y Baptista (2010: 78); cuyo diseño es:

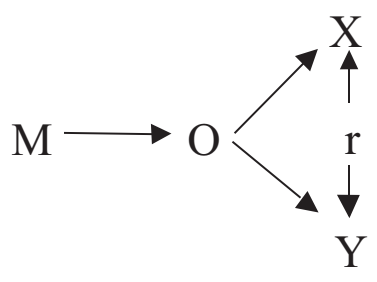

Donde

M: Muestra

$\mathrm{X}$ : Estado nutricional

Y: Creatividad

O: Observación

r: Correlación entre X e Y

Población, muestra y muestreo

\section{Población}

La población estuvo conformada por 30 estudiantes cuyas edades oscilan entre los 8 y 12 años, matriculados durante el año 2017, en la institución educativa $\mathrm{N}^{\circ} 17851$ de la comunidad Awajún de Chapi, provincia de Condorcanqui.

\section{Muestra y muestreo}

Se utilizó el muestreo no probabilístico, intencional o por conveniencia y estuvo conformado por 30 estudiantes, cuyas edades oscilan entre los 8 y 12 años, matriculados durante el año 2017, en la institución educativa $\mathrm{N}^{\circ} 17851$ de la comunidad Awajún de Chapi, provincia de Condorcanqui.

\section{Métodos, técnicas y procedimientos}

Métodos. los métodos que se utilizaron en la investigación son los siguientes:

Inductivo, mediante este método, fue posible describir y explicar la realidad de objeto de estudio asociado al estado nutricional y la creatividad, teniendo en cuenta el análisis del fenómeno estudiado, desde lo particular a lo general.

Deductivo, con este método se conoció la realidad global del problema, lo que sucedió en el mundo y relacionarlo con el objeto de estudio, a través del planteamiento del problema y los antecedentes. Se analizó el problema desde una visión holística hacia las particularidades sobre el estado nutricional y la creatividad de los estudiantes.

Analítico, este método nos facilitó conocer los resultados obtenidos mediante los instrumentos de recolección de datos y procesarlos en función a las variables de estudio.

Sintético, método que nos permitió construir las conclusiones a partir del análisis de los resultados en función a las variables de estudio.

\section{Técnicas e instrumentos}

Se utilizó la técnica de la observación, el cuestionario y el análisis documental y como instrumentos se utilizaron la prueba de imaginación creativa - PIC y la Tabla de valoración nutricional antropométrica para determinar el índice de masa corporal - IMC y el diagnóstico de talla para la edad, como se detalla a continuación.

\begin{tabular}{|l|l|}
\hline \multicolumn{1}{|c|}{$\begin{array}{c}\text { Técnicas de } \\
\text { investigación }\end{array}$} & \multicolumn{1}{|c|}{$\begin{array}{c}\text { Instrumentos de } \\
\text { investigación }\end{array}$} \\
\hline Observación & $\begin{array}{l}\text { Guía de observación, } \\
\text { libreta de apuntes } \\
\text { Tabla de valoración } \\
\text { nutricional } \\
\text { Tabla de diagnóstico de } \\
\text { talla para la edad }\end{array}$ \\
\hline Cuestionario & $\begin{array}{l}\text { Prueba de imaginación } \\
\text { creativa }\end{array}$ \\
\hline $\begin{array}{l}\text { Análisis } \\
\text { documental }\end{array}$ & Fichas de investigación \\
\hline
\end{tabular}

\section{Metodología}

Para realizar la presente investigación, se inició realizando las coordinaciones con el Apu de la comunidad nativa y el director de la Institución Educativa, en seguida se visitó al plantel para conocer a los estudiantes sometidos al estudio, realizándose una observación analítico - reflexiva y solicitar al órgano de dirección, algunos documentos de escolaridad de los alumno para implementar tablas de datos, luego midió el índice de masa corporal y el diagnóstico de talla para la edad, en una siguiente visita se administró la Prueba de imaginación creativa - PIC y finalmente se procesaron los datos obtenidos, utilizando programas estadísticos.).

\section{RESULTADOS}

Antes de presentar los resultados preliminares de la presente investigación, vamos a describir las 
dimensiones que comprende la prueba de imaginación creativa - PIC, teniendo en cuenta los sub test.

Tabla 1. Dimensiones e indicadores que comprende la prueba PIC.

\begin{tabular}{|c|c|c|}
\hline Prueba & Dimensión & Indicadores \\
\hline \multirow[b]{2}{*}{ Creatividad } & $\begin{array}{l}\text { Creatividad } \\
\text { narrativa }\end{array}$ & $\begin{array}{l}\text { Fluidez } \\
\text { Flexibilidad } \\
\text { Originalidad }\end{array}$ \\
\hline & $\begin{array}{l}\text { Creatividad } \\
\text { gráfica }\end{array}$ & $\begin{array}{l}\text { Originalidad } \\
\text { Elaboración } \\
\text { Sombras y color } \\
\text { Título } \\
\text { Detalles } \\
\text { especiales }\end{array}$ \\
\hline
\end{tabular}

Fuente: TEA ediciones S.A.

Tabla 2. Distribución de los estudiantes que forman parte de la muestra, por grados de estudio.

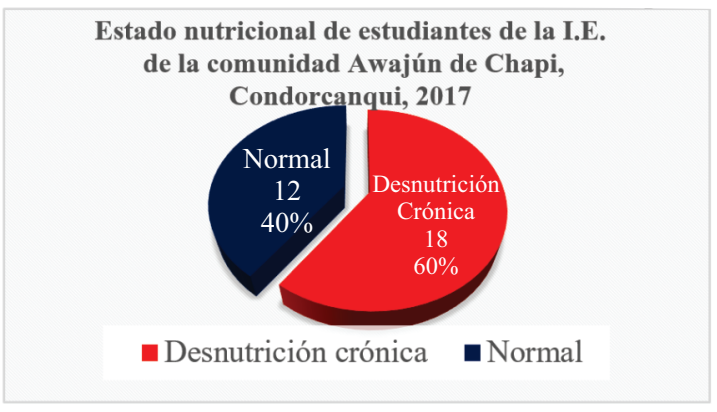

\begin{tabular}{|l|r|r|r|}
\hline \multirow{2}{*}{ Grado } & \multicolumn{2}{|c|}{ Género } & \multicolumn{1}{c|}{$\begin{array}{c}\text { Sub } \\
\text { total }\end{array}$} \\
\cline { 2 - 3 } & Femenino & \multicolumn{1}{|c|}{ Masculino } & \multicolumn{1}{c|}{ Tercero } \\
\hline Cuarto & 5 & 4 & 9 \\
\hline Quinto & 3 & 3 & 6 \\
\hline Sexto & 3 & 3 & 7 \\
\hline Total & 15 & 5 & 8 \\
\hline
\end{tabular}

Fuente: Nómina de matrícula de los estudiantes

Tabla 3, estado nutricional de los estudiantes de la institución educativa $\mathrm{N}^{\circ} 17851$ de la comunidad nativa de Chapi, Condorcanqui, 2017.

\begin{tabular}{ccc}
\hline Estado nutricional & $\mathrm{f}$ & $\%$ \\
\hline Desnutrición & 18 & 60 \\
crónica & 12 & 40 \\
Normal & 30 & 10 \\
\hline Total & & 0 \\
\hline
\end{tabular}

Fuente: Tabla de valor nutricional - Minsa

En la tabla 3 se observa que, de 30 niños y niñas sometidos al estudio, el $60 \%$ de ellos cuyas edades oscilan entre 8 y 12 años, se encuentran en estado de desnutrición crónica y solamente el $40 \%$ se encuentran en estado nutricional normal.

En la figura 1 se aprecia que el $60 \%$ es decir 18 estudiantes de un total de 30 comprendidos entre los 8 y 12 años de edad, matriculados en la institución educativa $\mathrm{N}^{\circ} 17851$ de la comunidad nativa de Chapi se encuentran en un estado de desnutrición crónica, y solamente 12 estudiantes equivalente al $40 \%$, su estado nutricional es normal.

En la figura 1 se aprecia que el $60 \%$ es decir 18 estudiantes de un total de 30 comprendidos entre los 8 y 12 años de edad, matriculados en la institución educativa $\mathrm{N}^{\circ} 17851$ de la comunidad nativa de Chapi se encuentran en un estado de desnutrición crónica, y solamente 12 estudiantes equivalente al $40 \%$, su estado nutricional es normal.

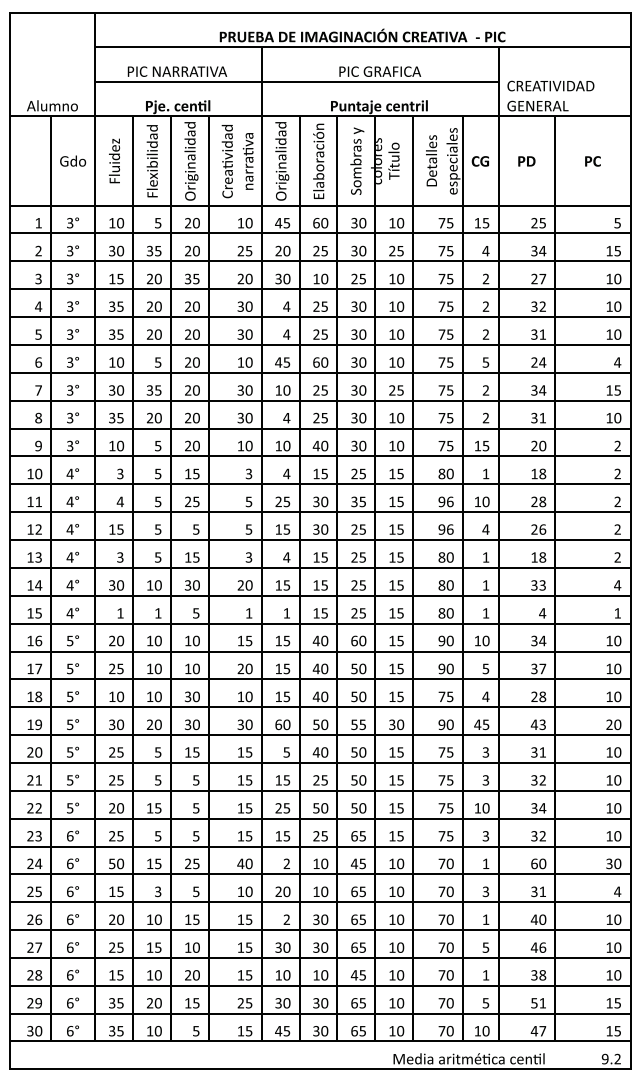


En la tabla 4, relacionada con los resultados de la prueba de imaginación creativa a los estudiantes comprendidos entre 8 y 12 años sometidos al estudio; después de haber procesado los resultados de las dimensiones de creatividad narrativa que comprende los indicadores de fluidez, flexibilidad y originalidad; y, creatividad gráfica que comprende los indicadores de originalidad, elaboración, sombras y colores, título y detalles especiales; se aprecia que la creatividad general centil, alcanza una media centil de 9 , observando además que solamente en tercero y sexto grado, en algunos indicadores pocos estudiantes obtienen un puntaje centil máximo de 15 , y solamente un niño del quinto grado alcanza un puntaje centil de 20; en términos generales, se concluye que, el nivel de creatividad de los niños sometidos a los niños y niñas es muy deficiente, situación preocupante en la educación básica de las comunidades Awajún.

Tabla 5. Correlaciones entre estado nutricional y creatividad de eatudiantes de la I.E. Condorcangui. 2017

\begin{tabular}{|c|c|c|c|}
\hline & & Nutrición & PC CREAGEN \\
\hline \multirow[t]{3}{*}{ Nutrición } & $\begin{array}{l}\text { Correlación } \\
\text { de Pearson }\end{array}$ & 1 & 076 \\
\hline & $\begin{array}{l}\text { Sig. } \\
\text { (unilatergl) }\end{array}$ & & ,345 \\
\hline & $\mathrm{N}$ & 30 & 30 \\
\hline \multirow{3}{*}{$\begin{array}{l}\text { Creatividad } \\
\text { General } \\
\text { PC }\end{array}$} & $\begin{array}{l}\text { Correlación } \\
\text { de Pearson }\end{array}$ & ,076 & 1 \\
\hline & $\begin{array}{l}\text { Sig. } \\
\text { (unilatergl) }\end{array}$ & ,345 & \\
\hline & $\mathrm{N}$ & 30 & 30 \\
\hline
\end{tabular}

Fuente: Resultados de las pruebas de nutrición y de creatividad, 2017

En la tabla 5, están los resultados de la correlación entre el estado nutricional y la prueba de imaginación creativa de los estudiantes sometidos al estudio, se observa que no existe correlación directa significativa entre el estado nutricional y la creatividad de los niños y niñas de 8 a 12 años que formaron parte de la muestra.

En la figura 2 se muestra la correlación entre estado nutricional y creatividad general de los estudiantes, los resultados obtenidos demuestran que no existe correlación directa entre estas dos variables de estudio.

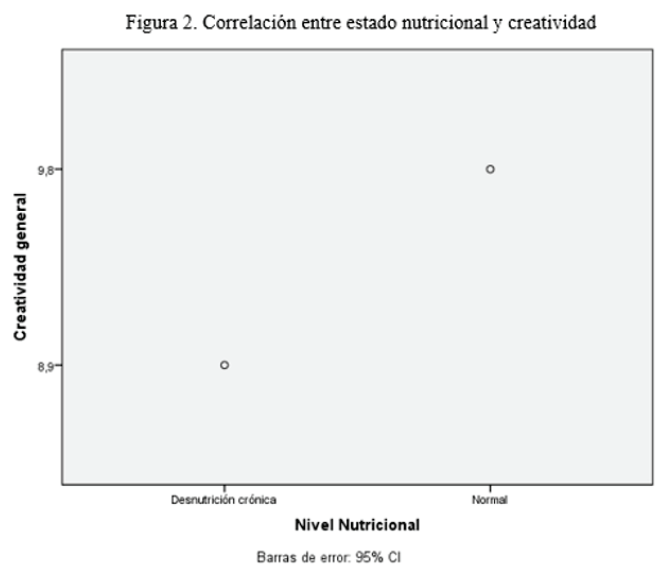

\section{DISCUSIÓN}

La tasa de desnutrición de la muestra sometida al estudio, es alta dado que el $60 \%$ de estudiantes presentan un estado de desnutrición crónica y solamente el $40 \%$ se encuentra en estado de desnutrición normal. Resultado que coincide con los hallazgos Ortiz, Peña y Albino, quien, en su trabajo de investigación sobre Desnutrición infantil, salud y pobreza: intervención desde un programa integral. Trabajo realizado entre un equipo multidisciplinario que asocia a la Universidad Diego Portales de Santiago de Chile; Universidad Las Palmas de Gran Canaria, España, Hospital universitario materno infantil de Canarias y la Fundación Corporación para la nutrición infantil - CONIN; concluye que la atención preventiva en la desnutrición es clave, situación que no se da en la comunidad nativa, las autoridades descuidan a la niñez en todos los aspectos.

En lo que respecta a la tasa de creatividad, donde se han considerado la dimensión de creatividad narrativa con sus indicadores de fluidez, flexibilidad, originalidad y la dimensión de creatividad gráfica, con sus indicadores de originalidad, elaboración, sombras y color, título y detalles especiales; se aprecia que los estudiantes sometidos al estudio, alcanza una media centil de 9, observando además que solamente en tercero y sexto grado, en algunos indicadores pocos estudiantes obtienen un puntaje centil máximo de 15 , y solamente un niño del quinto grado alcanza un puntaje centil de 20; en términos generales, se concluye que, el nivel de creatividad de los niños sometidos a los niños y niñas es muy deficiente. Resultado que coincide con la investigación de Matalinares, sobre "Efectos del déficit nutricional en la creatividad de alumnos de educación primaria", quien concluye que la creatividad, en sus diferentes dimensiones e indicadores (fluidez, flexibilidad, organización y originalidad), narrativa y gráfica, no siguen un patrón de desarrollo uniforme en función de la edad ni del grado de instrucción. Muy por el contrario, se han revelado oscilaciones e incluso caídas en los puntajes del último grado de instrucción y del grupo de mayor edad estudiado. Lo cual hace plausible pensar que el tener mayor edad o mayor grado de instrucción, no necesariamente significa ser más creativo y pone al tapete la propuesta de Torrance, quien plantea que hay etapas en que la educación pone más frenos al desarrollo de la creatividad.

En lo que respecta a la correlación entre el estado nutricional y la creatividad de los estudiantes Awajún comprendidos entre los ocho y doce años, los 
resultados muestran que no existe correlación directa significativa, sin embargo en la correlación entre creatividad general y grado de estudios se muestra una correlación entre el grado de estudios y creatividad, es decir conforma avanzan en su grado de estudios se incrementa levemente su creatividad, a excepción del cuarto grado donde los niños descienden significativamente en la media de su creatividad centil.

\section{CONCLUSIONES}

De la ejecución del presente trabajo de investigación, se puede concluir en lo siguiente:

La tasa de desnutrición de la muestra sometida al estudio, es alta dado que el $60 \%$ de estudiantes tienen un estado de desnutrición crónica y solamente el $40 \%$ se encuentra en estado de desnutrición normal.

En lo que respecta a la creatividad de los niños y niñas comprendidas entre los ocho y 12 años, que formaron parte a la muestra, en la dimensión de creatividad narrativa con sus indicadores de fluidez, flexibilidad, originalidad y la dimensión de creatividad gráfica, con sus indicadores de originalidad, elaboración, sombras y color, título y detalles especiales, se aprecia que tienen un bajo nivel de creatividad, tal es así que alcanzan una media centil de 9 .

La correlación entre el estado nutricional y la creatividad de los estudiantes Awajún comprendidos entre los ocho y doce años, los resultados muestran que no existe correlación directa significativa, sin embargo en la correlación entre creatividad general y grado de estudios se muestra una correlación entre el grado de estudios y creatividad, es decir conforma avanzan en su grado de estudios se incrementa levemente su creatividad, a excepción del cuarto grado donde los niños descienden significativamente en la media de su creatividad centil.

\section{REFERENCIAS BIBLIOGRÁFICAS}

Bermejo, R., Hernández, D., Ferrando, M., Sainz, M. y Prieto, D. (2010). Creatividad, inteligencia sintética yalta habilidad. [artículoen línea]. $\mathrm{R}$ e c u p e r a d o dehttp://ezproxy.concytec.gob.pe:2161/Cont entServer.asp? $\mathrm{T}=\mathrm{P} \& \mathrm{P}=\mathrm{AN} \& \mathrm{~K}=51503625 \&$ $\mathrm{S}=\mathrm{R} \& \mathrm{D}=$ fua $\&$ EbscoContent $=\mathrm{dGJyMNHr} 7$ ESeprQ4y9fwOLCmr06ep7VSsaa4TK6Wx
WXS\&ContentCustomer $=\mathrm{dGJyMPGvsEq0r}$ q5MuePfgeyx44Dt6fIA

Cemades, I. (2008). Desarrollo de la creatividad en educación infantil; Revista creatividad y sociedad. $\mathrm{N}^{\circ}$. 12. [artículo en línea]. Recuperadodehttp://www.creatividadysocied ad.com/articulos/12/Creatividad $\% 20 \mathrm{y} \% 20 \mathrm{~S}$ ociedad. $\% 20$ Desarrollo $\% 20 \mathrm{de} \% 201 \mathrm{a} \% 20$ cre atividad $\% 20$ en $\% 20$ Educacion $\% 20$ Infantil.p df

Hernández, Fernández y Baptista. (2013). Metodología de la investigación científica”. Ciudad de México: MacGrawHill.

Fuentes, R. (2004). Desarrollar la creatividad desde los contextos educativos: un marco de reflexión sobre la mejora socio-personal; Revista electrónica iberoamericana sobre calidad, eficacia y cambio de educación REICE. Vol. 2, $\mathrm{N}^{\circ}$ 1. [artículo en línea]. $R$ e c u p e r a https://repositorio.uam.es/bitstream/handle/1 0486/660709/REICE_2_1_10.pdf?sequence $=1$

Instituto de estudios peruanos - UNICEF (2009). Programa Juntos: certezas y malentendidos en torno a las transferencias condicionadas - Estudio de caso de seis distritos rurales del Perú-. Recuperado el 6 de marzo de 2016, d e $\quad$ s $\quad$ d e e file://C:/Users/CyberPlanet_Estandar/Down loads/huber_programajuntos.pdf.

Matalinares, M. (2004). Efectos del déficit nutricional en la creatividad de alumnos de educación primaria (Tesis Doctoral). Universidad Nacional Mayor de San Marcos. Lima.

Mejía, E. (2015). Metodología de la investigación cientifica. (3era. Ed.). Lima: San Marcos.

MIDIS - ENDES (2016). Reporte desnutrición crónica infantil; Encuesta demográfica y de salud familiar-ENDES, 2012. Recuperado el $06 \mathrm{de}$ marzo de 2016 , desde www.midis.gob.pe/index.php/es/direcciongeneral-de-politicas-y-estrategias/reportedesnutricion-cronica-infantil-meta-2016. 
Naciones Unidas - CEPAL (2010). La hora de la igualdad: brechas por cerrar, caminos por abrir. Santiago de Chile.

Ortiz, A. Peña, L. Albino, A., Monckerberg, B. \& Serra, L. (2006). Desnutrición infantil, salud y pobreza: intervención desde un programa integral (Tesis que asocia a equipo multidisciplinario de instituciones). Universidad Diego Portales, Chile; Universidad de Gran Canaria, España; Hospital universitario materno infantil de Canarias; Fundación Corporación para la nutrición infantil - CONIN). Mendoza, Argentina.

Segura, L. Montes, C. Hilario, E. Asenjo, P. y Baltazar, G. (2002). Pobreza y desnutrición infantil. Recuperado el 05 de marzo de 2016, desdehttps://www.mef.gob.pe/contenidos/po 1_econ/documentos/Pobreza_y_Desnutricio n.pdf

TEAediciones (2017). Prueba de imaginación creativa - TEAediciones S.A. Madrid: imprenta de TEAediciones.

UNICEF (2016). Una oportunidad para cada niño: estado mundial de la infancia. Fondo de las Naciones Unidas para la Infancia. Nueva York.

UNICEF (2011). La desnutrición infantil Causas, consecuencias y estrategias para su prevención y tratamiento. Recuperado el 05 $\mathrm{de} \mathrm{marzo}$ d e 2016 , d e s d e https://www.unicef.es/sites/www.unicef.es/fi les/Dossierdesnutricion.pdf

UNICEF, (2012). Desnutrición crónica infantil cero en el 2016: una meta alcanzable; Resumen ejecutivo. Recuperado el 6 de marzo de $\begin{array}{llllllllll}2 & 0 & 1 & 6 & , & \mathrm{~d} & \mathrm{e} & \mathrm{s} & \mathrm{d} & \mathrm{e}\end{array}$ http://inversionenlainfancia.net/infobaromet ro/boletines/informe_desnutricion_resumen. pdf 